\title{
The Bulky Dermoid Cyst of the Floor of the Mouth
}

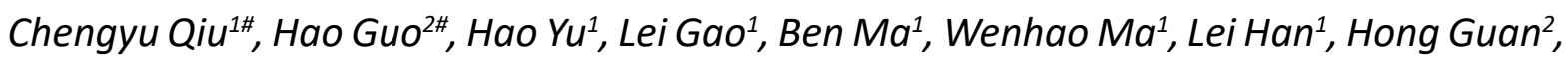 \\ Zhihui Sun ${ }^{1 *}$, Hong Chen ${ }^{1 *}$ \\ ${ }^{1}$ The First Hospital of Qiqihaer City, Qiqihaer, Heilongjiang, China \\ ${ }^{2}$ Research Center of Microecological Engineering Technology, Qiqihaer Medical University, Qiqihaer, Heilongjiang, \\ China
}

${ }^{\#}$ These Authors contributed equally to this work.

\begin{abstract}
Bulky dermoid cysts of the floor of the mouth are very rare and may induce functional disorder. We present a 68-year-old Chinese woman who presented a painless swelling in right mandibular. Physical examination revealed a painless, soft, poor mobility, dough like mass swelling which was reached to the floor of the mouth, and MRI showed a circumscribed mass of about $20.0 \mathrm{~cm} \times 10.0 \mathrm{~cm} \times 12.0 \mathrm{~cm}$ size. After complete excision of the cyst through an extraoral approach, histology diagnosed dermoid cyst. Dermoid cyst is uncommon found in the floor of the mouth, where there is a soft, painless, associated dyspnea, dysphagia and dysarthria. Imaging may assist diagnosis. Definitive diagnosis is founded on the histology specimen. According to the location and size of the cyst on each occasion, enucleation via intraoral or extraoral approach will be chosen treatment for cyst in the floor of the mouth. Although malignant degeneration of dermoid cyst of the floor of mouth is extremely rare, there are also such a situation reports. Thus, it is important to remove a cyst before the opportunity for malignancy.
\end{abstract}

\section{Keywords}

Cyst, Dermoid Cysts, Floor of The Mouth

\section{Introduction}

Dermoid cysts (DC) are benign congenital hamartomas of ectodermal origin that can found in any region of the body [1]. Research showed that nearly $7 \%$ occurred in the head and neck area [1], and only $6 \%$ are found laterally and present in the submandibular region [2]. A dermoid cyst is a benign neoplasm that is derived from both ectoderm and mesoderm [3]. The benign neoplasm is typically asymptomatic, huge, soft, slow-growing and painless, making clinical distinction difficult. To delineate the location, magnetic resonance imaging (MRI) is the first choice for imaging evaluation. And the final diagnosis of a DC depends on histological investigation. We report the case of a patient with a dermoid cyst in the floor of the mouth and note the MRI findings. It appeared to the surgical excision is the treatment of choice.

\section{Case Report}

A 68-year-old Chinese woman referred to the Department of Oral and Maxillofacial Surgery, the First Hospital of Qiqihaer City with a chief complaint of a painless swelling in right mandibular (Figure 1). Forty years earlier, she had first noticed painless swelling in the right side of the neck, which was not associated with any pain or any discharges. Due to the swelling increased slowly, the patient neglected the examination at that time. Recently, the patient noticed that the swelling grew fast, which extended from the right submandibular region to the submental and the left region. No visible facial deformity was observed. Physical examination revealed a painless, soft, poor mobility, dough like mass swelling which was reached to the floor of the mouth, below the root of the neck, around bilateral sternocleidomastoid zone. The overlying skin of the mass swelling and the colour of the oral mucosa appeared normal. There was no history of any previous operative procedure. No history of noisy breathing. No other abnormalities were detected in general examinations.

To delineate the location and quality of the swelling, magnetic resonance imaging (MRI) was carried out. MRI showed a circumscribed mass of about $20.0 \mathrm{~cm} \times 10.0 \mathrm{~cm}$

*Corresponding author: Zhihui Sun and Hong Chen, The First Hospital of Qiqihaer City, Qiqihaer, Heilongjiang, China

Accepted: July 15, 2021

Published online: July 17, 2021

Citation: Qiu C, Guo H, Yu H, et al. (2021) The Bulky Dermoid Cyst of the Floor of the Mouth. J Head Neck Surg 3(1):157-159 
$\times 12.0 \mathrm{~cm}$ size extended to the front of masseter muscle, beneath the floor of the mouth and on top of the thyroid, next to the carotid sheath, along with compression of the trachea (Figure 2). A complete surgical enucleation under general anesthesia was performed.

During the operation, the swelling was found at the floor of the mouth muscle group. The swelling was exposed by cutting off the bottom of the mouth muscles. Following it was completely excised by means of a blunt dissection instrument (Figure 3). The surgical wound was rinsed with normal saline solution and bio-fluid film. Bleeding was about $100 \mathrm{~mL}$ and was controlled, and the wound was sutured with an absorbable suture material. The macroscopic appearance of the enucleated specimen was cystic in nature, and it was filled with a mixture of serous liquid and paste-like materials that were yellowish white (Figure 4). The excised specimen was sent for histopathologic examination. Microscopic examination showed a cystic capsule lined by stratified squamous epithelium and a connective tissue capsule with the skin glands (Figure 5).

\section{Discussion}

Dermoid cysts in the mouth account for less then $0.01 \%$ of all oral cysts. Among the cases reported, several dermoid cysts that exist at submental or sublingual space, but a large dermoid cyst like our case was not reported. A previous

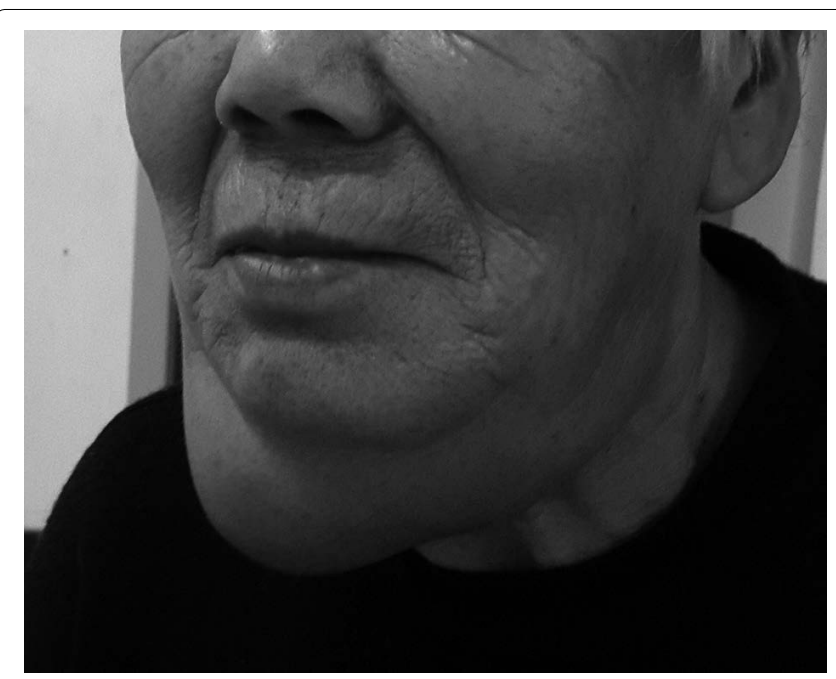

Figure 1: The enormous dermoid cyst at the floor of the mouth.

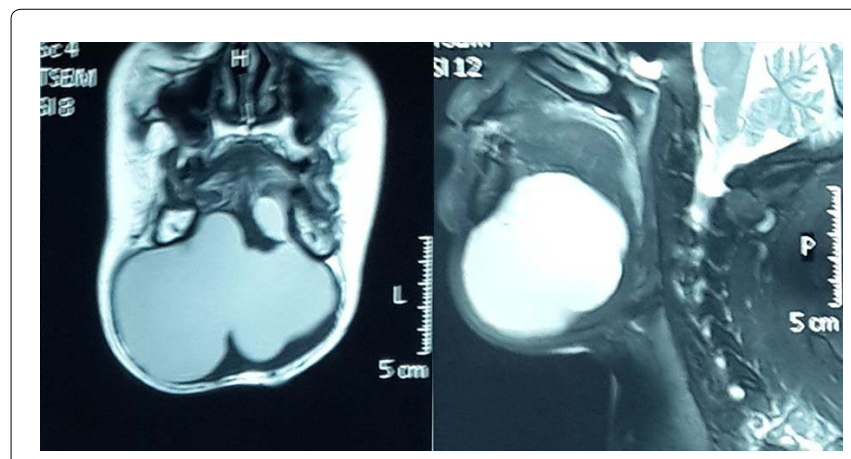

Figure 2: MRI imaging of floor of the mouth dermoid cyst.

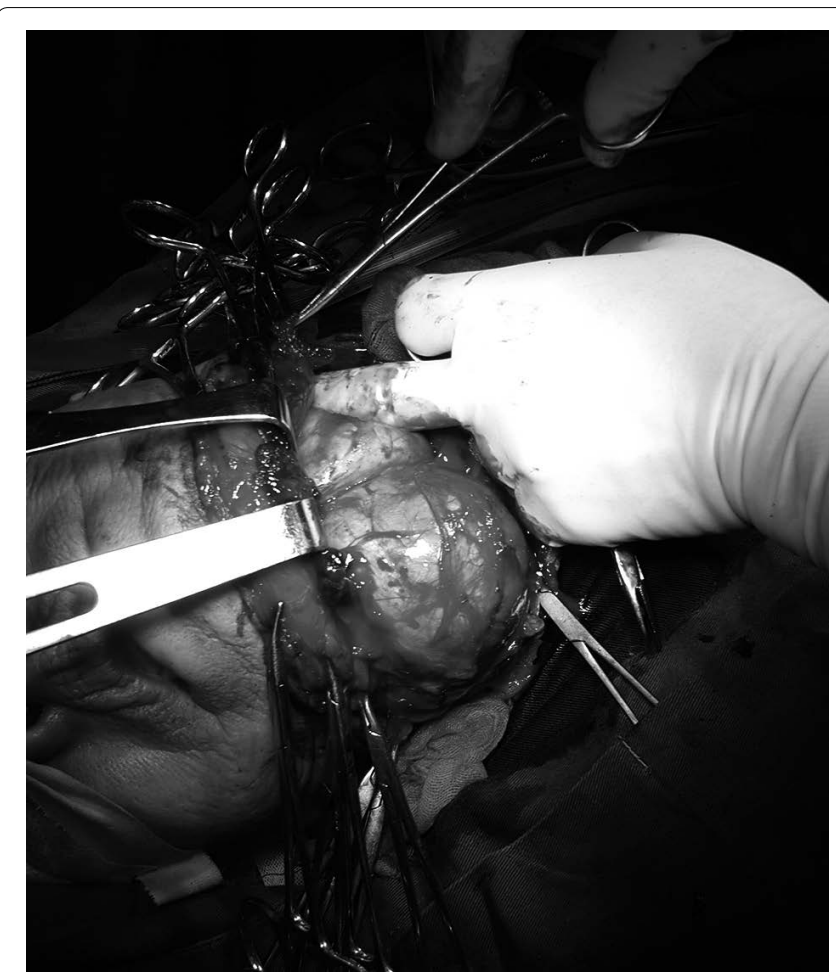

Figure 3: Intraoperative view. The cyst can be resected completely.

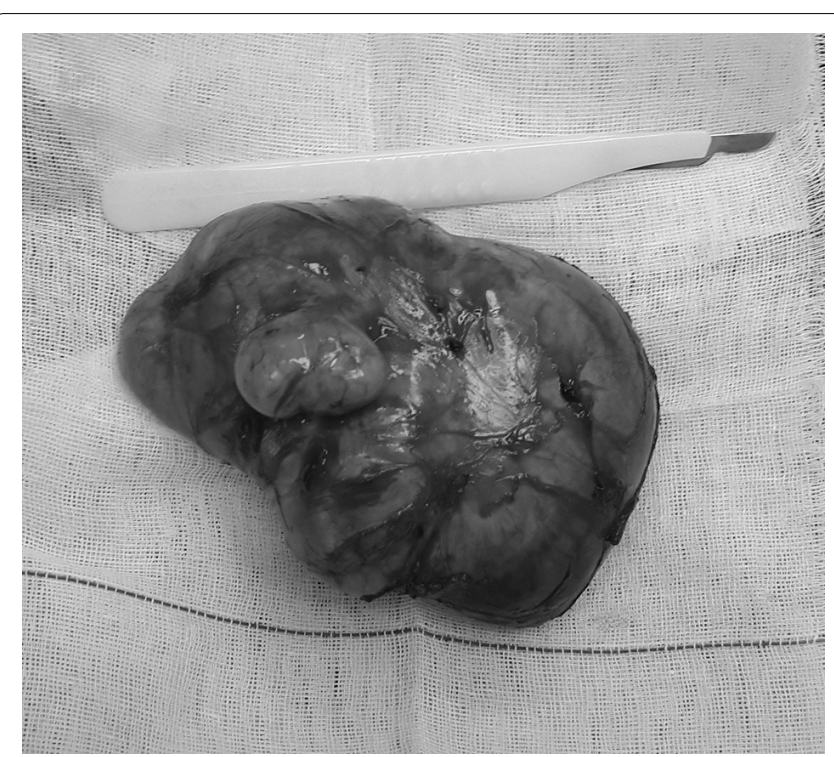

Figure 4: Macroscopic view of excised specimen.

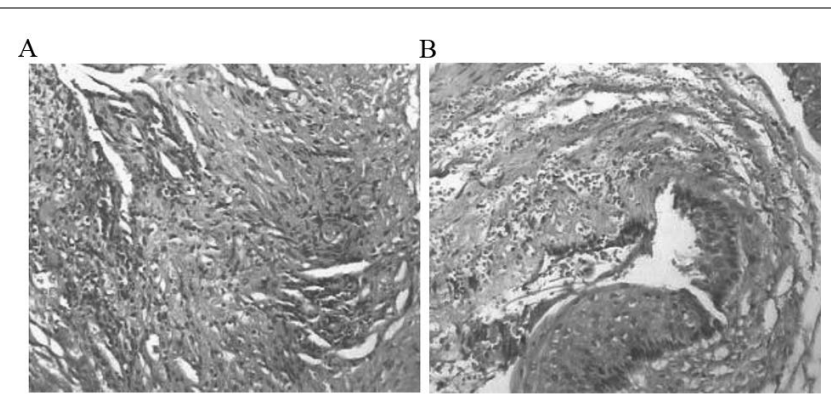

Figure 5: Histopathological appearance of dermoid cyst (HE $\times$ 100). 
case study reported a 77-year-old Japanese female who noted a cyst over a period of ten years, however, its size was only $11 \mathrm{~cm} \times 9 \mathrm{~cm} \times 9 \mathrm{~cm} \mathrm{[4].} \mathrm{In} \mathrm{our} \mathrm{case,} \mathrm{the} \mathrm{patient} \mathrm{had}$ noticed the swelling about 40 years prior, but did not seek medical attention. Over the time, the swelling enlarged, and eventually, made speaking difficult and narrowed the airway. When considering its final size, we thought that she should have sought medical attention earlier.

According to histological findings or their location, dermoid cyst in the floor of the mouth is classified as three types: Epidermoid cyst, dermoid cyst and teratoid cyst $[2,5]$. The essential difference between dermoid cyst and epidermoid cyst is the skin appendages exist under the cyst wall, and dermoid cyst is more frequent than epidermoid cyst at a ratio of 2: 1 . A teratoid cyst contains other major organ systems organization. Dermoid cyst is uncommon tumor found in the floor of the mouth [6], where there is a soft, painless, associated dyspnea, dysphagia and dysarthria [7]. If the cyst develops in the submental area, which sometimes raises the floor of the mouth, a double chin will appear [8]. The appearance of these cysts is most frequently reported in adults in their 20's and 30's [9], as the first time for patients in our case to be at the age of 20.

Imaging can assist diagnosis [10]. Magnetic resonance imaging (MRI) is an accurate, noninvasive modality for the diagnosis and is valuable to distinguish the cyst for a provisional diagnosis. It has the advantage to delineate the cyst and demonstrate the exact extension through the floor of the mouth and the internal architecture. In our case, MRI was superior to other imaging modalities in distinguishing between cystic and solid masses. And it could also guide the choice of surgical approach. According to the location and size of the cyst on each occasion, enucleation via intraoral or extraoral approach will be chosen treatment for cyst in the floor of the mouth. Generally speaking, the intraoral approach is best suited to small cyst, and when the cyst is so large or extending under the geniohyoid muscle or the mylohyoid muscle, an extraoral approach is required [11]. An extraoral approach, as in the present case, was the effective treatment whereas the patient had the symptoms include dysphagia and dyspnea, and the cyst was so large in the submental and submandibular spaces. Although malignant degeneration of dermoid cyst of the floor of mouth is extremely rare, there are also such a situation reports. Thus, it is important to remove a cyst before the opportunity for malignancy.

\section{Conclusion}

In conclusion, dermoid cyst in the floor of the mouth surviving so long is quite rare. When bulky, they may induce functional disorder. Furthermore, MRI describes a detailed examination of its size and anatomical location, and provides the surgical operation treatment. The ultimate definite diagnosis of the disease is always affected via histopathological examination.

\section{Reference}

1. Kondo S, Kamatani T, Saito Y, et al. (2015) True lateral dermoid cyst of the submandibular region: A case report. Journal of Oral and Maxillofacial Surgery, Medicine, and Pathology 27: 74-77.

2. Teszler CB, El-Naaj IA, Emodi O, et al. (2007) Dermoid cysts of the lateral floor of the mouth: A comprehensive anatomosurgical classification of cysts of the oral floor. Journal of Oral and Maxillofacial Surgery 65: 327-332.

3. Pryor SG, Lewis JE, Weaver AL, et al. (2005) Pediatric dermoid cysts of the head and neck. Otolaryngol Head Neck Surg 132: 938-942.

4. Isomura ET, Matsuoka Y, Hamaguchi M, et al. (2011) Large epidermoid cyst in lateral floor of mouth and submandibular region. Asian Journal of Oral and Maxillofacial Surgery 23: 99101.

5. RC King, BR Smith, JL Burk (1994) Dermoid cyst in the floor of the mouth. Review of the literature and case reports. Oral Surg Oral Med Oral Pathol 78: 567-576.

6. Irving Meyer (1955) Dermoid cysts (dermoids) of the floor of the mouth. Oral Surgery, Oral Medicine, Oral Pathology 8: 1149-1164.

7. Seward BSGR (1965) Dermoid cysts of the floor of the mouth. Br J Oral Surg 3: 36-47.

8. Longo F, Maremonti P, Mangone G M, et al. (2003) Midline (Dermoid) cysts of the floor of the mouth: Report of 16 cases and review of surgical techniques. Plast Reconstr Surg 112: 1560-1565.

9. Oatis GW Jr, Hartmen GL, Robertson GR, et al. (1975) Dermoid cyst of the floor of the mouth. Report of a case. Oral Surg Oral Med Oral Pathol 39: 192-198.

10. E Bokoa, Amaglob K, Kpemissia E (2014) A bulky dermoid cyst of the floor of the mouth. Eur Ann Otorhinolaryngol Head Neck Dis 131: 131-134.

11. Turki IM, Mouaffak-Zidi Y, Rajhi H, et al. (2011) A case of large dermoid cyst of the tongue. Egyptian Journal of Ear, Nose, Throat and Allied Sciences 12: 171-174.

DOI: $10.36959 / 605 / 560$

Copyright: (C) 2021 Qiu C. This is an open-access article distributed under the terms of the Creative Commons Attribution License, which permits unrestricted use, distribution, and reproduction in any medium, provided the original author and source are credited. 